Beyond the Classical Instability Strip 


\title{
New Opacities and the Beta Cephei Stars
}

\author{
L.A. Balona \\ South African Astronomical Observatory, P.O.Box 9, Observatory 7985, Cape, \\ South Africa
}

\begin{abstract}
The recent revision of metal opacities has opened up the possibility that the long-sought mechanism for driving pulsations in these stars has at last. been found. This hypothesis makes a testable prediction - that no $\beta$ Cep variables should exist among metal-poor B-type stars. We report on the results of an intensive CCD monitoring campaign to test this prediction. The question of the pulsation mode of $\beta$ Cep stars and the consequence of a revision of the absolute magnitudes to accommodate the fundamental radial mode among these stars is also discussed. Pulsational instability due to driving by ionization of metals has many other repercussions for the incidence of pulsation among the B-type stars. A classification scheme for other intrinsically variable Btype stars is suggested. It is shown that if, as generally supposed, pulsation is common among B-type stars then at least two different mechanisms must be in operation.
\end{abstract}

\section{Introduction}

The $\beta$ Cep stars were first recognized nearly a century ago by their short-period radial velocity variations and light variations. The periods (generally 3 to 6 hours) are too short to be ascribed to stellar rotation or binary interaction. The best definition of what constitutes a $\beta$ Cep star will ultimately depend on the physics of stellar pulsation in early-type stars. One definition is that a $\beta$ Cep star must have at least one radial pulsation mode. While this may yet prove to be a good definition, I am of the opinion that we do not yet know enough of the physics of the pulsation, of what constitutes a radial mode in rapidly-rotating stars and how one might identify modes (especially in faint stars) to make this definition very useful at the present time. The best definition for such a star still appears to be the one in general use for most of this century: an early B- or late O-type star with a radial velocity or photometric period too short to be explained by anything other than pulsation. In terms of pulsation theory, this merely implies a $p$-mode pulsation of low spherical harmonic order.

With the recent revision of metal opacities (Rozsnayi 1989, Iglesias \& Rogers 1991), the possibility now exists of understanding the mechanism which drives pulsations in the $\beta$ Cep stars (Cox \& Morgan 1990, Cox et al. 1992, Moskalik \& Dziembowski 1992, Kiriakidis et al. 1992). The sudden appearance of a tremendous 
number of iron lines at a temperature of about $150,000 \mathrm{~K}$ gives a high sensitivity of opacity to temperature at the very low densities found in these stars. In this paper we discuss the implications of this mechanism for $\beta$ Cep and other pulsating B stars.

\section{Dependence of driving on metal abundance}

The clearest prediction, and one in which there is agreement among all investigators, is the high sensitivity of the pulsations to metal abundance. Pulsation is found only for stars with a metal abundance somewhat larger than the solar value, but this depends very sensitively on the opacity and may change as the opacities are improved. Nevertheless, detection of $\beta$ Cep pulsations in a metal-poor star would pose a major problem.

The detection of a $\beta$ Cep star, PHL 346, situated at more than $5 \mathrm{kpc}$ from the galactic plane (Waelkens \& Rufener 1988) is of particular interest in this regard. Waelkens \& Rufener (1988) argue that the star could not have been formed in the galactic plane and belongs to Population II. However, there is no direct evidence that metals are underabundant in this star. More recently, Waelkens, Van den Abeele \& Van Winckel (1991) found that the $\beta$ Cep stars in the direction of the galactic centre seem to be hotter than normal, indicating a possible dependence on metal abundance. Again, the evidence is not very compelling.

\section{A search for $\beta$ Cep stars in LMC clusters}

The mechanism should cease to be effective when the metal abundance is sufficiently low. Therefore $\beta$ Cep stars should not exist in metal-poor systems such as the Magellanic Clouds. This was found to be the case in the cluster NGC 330 in the SMC (Balona 1992a). A large proportion of early B-type stars in NGC 330 are Be stars (Grebel, Richtler \& de Boer, 1992); indeed Balona (1992a) found a substantial number of periodic Be stars ( $\lambda$ Eri variables) in this cluster. The $\beta$ Cep phenomenon is rare among rapid rotators such as Be stars and in any case would be difficult to detect owing to the irregular light variations in these stars. Under the circumstances, the lack of $\beta$ Cep stars in NGC 330 is not too surprising and is probably not sufficient evidence in favour of the metal opacity mechanism.

With this in mind, Balona \& Jerzykiewicz (1992) and Balona (1992b) obtained intensive CCD photometry of two young clusters in the LMC: NGC 2004 and NGC 2100. These are not known to contain significant numbers of Be stars and no confirmed $\lambda$ Eri variables were detected. Over 150 observations for each star were obtained over a period of 12 nights. A total of 178 stars lying in the $\beta$ Cep instability strip were examined. The intrinsic colour-magnitude diagrams of the two clusters, together with the extreme magnitude limits of the $\beta$ Cep instability strip, are shown in Figs. 1 and 2.

Not a single star was found with the characteristic short-period variability, 


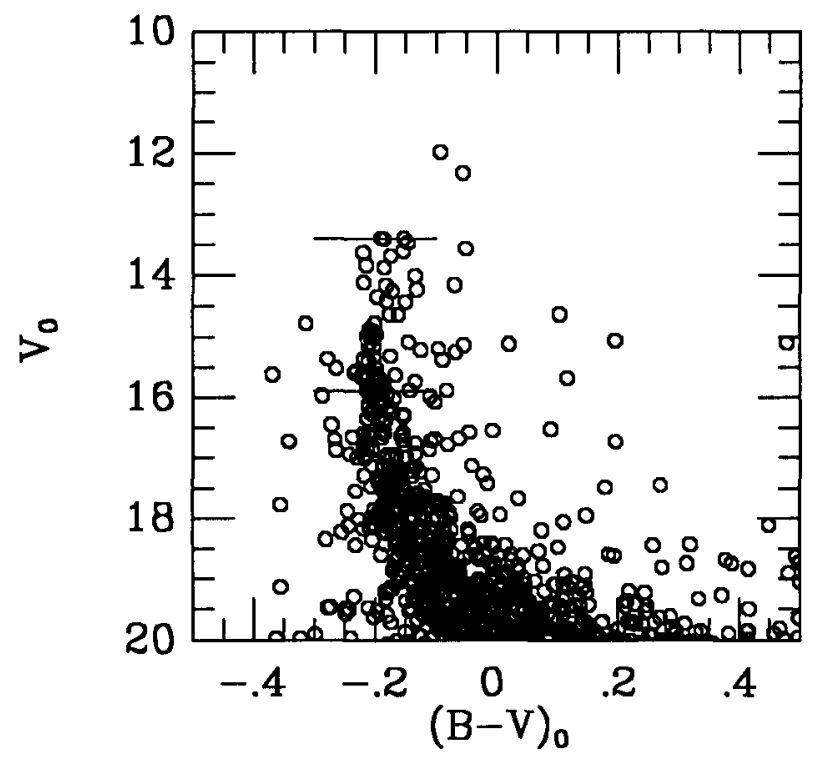

Figure 1 The intrinsic colour-magnitude diagram for the blue main sequence in the LMC cluster NGC 2004. The extreme magnitude limits of the $\beta$ Cep instability strip is shown by the two lines.

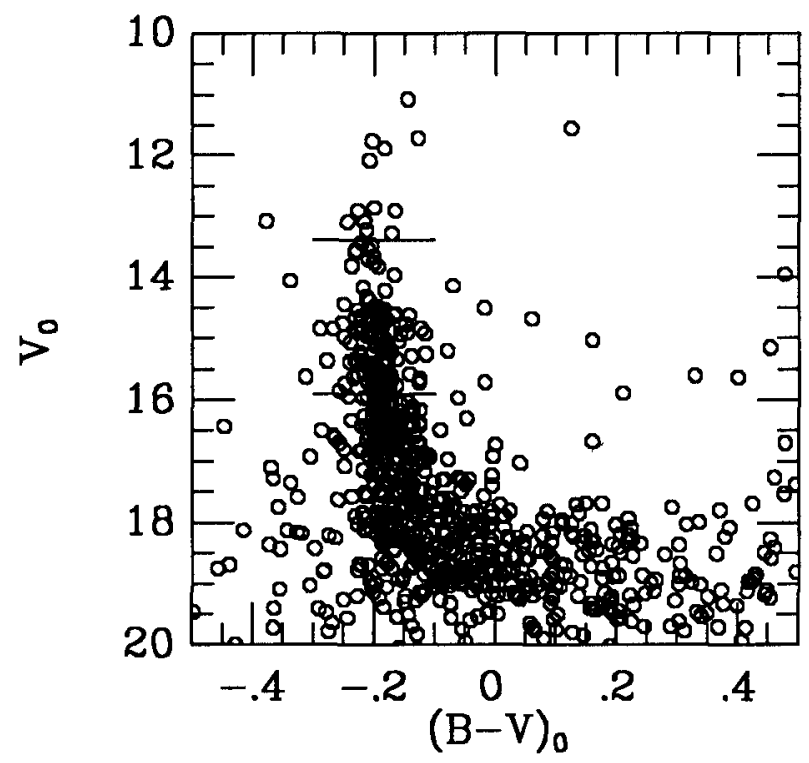

Figure 2 The intrinsic colour-magnitude diagram for the blue main sequence in the LMC cluster NGC 2100 . The extreme magnitude limits of the $\beta$ Cep instability strip is shown by the two lines. 
though the observations would have been able to detect such stars with amplitudes as small as $0.01 \mathrm{mag}$. The approximate proportion of B0-B2 main sequence and giants which are $\beta$ Cep variables can be estimated from the Bright Star Catalogue. There are 404 stars of this kind in the catalogue, of which 31 appear as definite $\beta$ Cep stars in the catalogue of Jerzykiewicz \& Sterken (1992). This gives a value of 7.5 percent for the expected proportion of $\beta$ Cep stars among galactic B0-B2 dwarfs and giants, but it must be taken as a lower limit because not all stars of this type in the Bright Star Catalogue have been surveyed for short-period variability. Certainly the proportion among Galactic open clusters is considerably higher.

On the assumption that the fraction of $\beta$ Cep stars in the two LMC clusters is the same as in the Galaxy, the chance of not finding a single variable in the 178 stars surveyed in NGC 2004 and NGC 2100 is less than 0.1 percent. This is very strong evidence in favour of a dependence on metallicity.

In spite of this strong support for the current theory, the possibility exists that the age of the clusters are not suitable for producing $\beta$ Cep pulsation. Although both NGC 2004 and NGC 2100 contain stars which are classified as B0-B2 dwarfs and giants and therefore should be good candidates for $\beta$ Cep variations, the clusters are actually older than galactic clusters such as NGC 3293 and NGC 4755 which also contain stars with these spectral types (Balona 1992b). The reason for this is the lower metallicity which has the effect of increasing the effective temperature at a given luminosity. It is just possible that the instability strip at low metal abundances may be at a different location from the one with normal metal abundances. It would be important to survey a younger LMC cluster to make sure that this is not the case.

\section{The mode of pulsation}

Moskalik \& Dziembowski (1992) and Kiriakidis et al (1992) both find that only fundamental $p$-modes are excited. Cox \& Morgan (1990) also find instability in one or two overtones, a result which may not be real (see Moskalik \& Dziembowski, 1992). A test for the mode of pulsation depends on an estimate for the pulsation constant, $Q$, which in turn depends mostly on knowing the absolute magnitude of the star. Most $\beta$ Cep stars are multiperiodic, so it is certain that non-radial modes are also excited. This complicates the issue because rotational splitting of these modes will give erroneous $Q$ values. Nevertheless, the mean $Q$-value of a group of stars may be taken to approximate the predominant pulsation mode.

Shobbrook (1983) found that the $\beta$ Cep stars in NGC 3293 were predominantly pulsating in the fundamental radial mode $(Q=0.034)$, in agreement with current theoretical predictions. However, this work was based on a preliminary calibration of the absolute magnitudes in terms of Strömgren indices which was subsequently revised by Balona and Shobbrook (1984). The revised calibration showed that 
for most $\beta$ Cep stars $Q=0.029$ which corresponds more closely with the first overtone radial mode (Shobbrook 1985). This is consistent with all other attempts at estimating this quantity (Balona \& Feast 1975, Shobbrook 1979, Jakate 1980). A detailed investigation of the pulsation modes in the $\beta$ Cep stars in NGC 3293 (Engelbrecht 1986) shows that most of these stars are overtone pulsators.

The estimation of $Q$ depends sensitively on the absolute magnitudes of the early B-type stars. It is important to investigate whether the current calibrations may not overestimate the luminosity of these stars by about $0.4 \mathrm{mag}$, which is required for fundamental radial pulsation. There is little doubt that the absolute magnitude calibration in terms of Strömgren indices is susceptible to systematic errors (Balona \& Shobbrook 1984). Millward \& Walker (1985) attempted to overcome these errors by using high signal-to-noise spectra to measure the $H \gamma$ equivalent width. No direct comparison was made between these absolute magnitudes and the Balona \& Shobbrook (1984) calibration, but the distance modulus for the one young star cluster in common, NGC 2244, is the same. This calibration is not likely to make a significant difference in the $Q$ values for $\beta$ Cep stars. Perhaps the most comprehensive investigation of this problem is the work of Wolff (1990) who used spectroscopic measurements of $H \gamma$ to re-calibrate the luminosities of the B-type stars. This calibration gives absolute magnitudes which are about $0.15 \mathrm{mag}$ fainter than those of Balona \& Shobbrook (1984) in the range appropriate to $\beta$ Cep stars. This is in the right direction, but too small to push the mean $Q$ value towards fundamental radial pulsation. My conclusion is that while there may well be systematic errors in the present absolute magnitude calibration of early B-type stars, they are unlikely to be as large as the 0.4 mag required to give a mean pulsation constant appropriate to the fundamental radial mode. A systematic error of this magnitude would, moreover, almost certainly affect the zero-point of the Cepheid distance scale and destroy the agreement now reached between pulsation and evolution masses for these stars.

\section{Some outstanding problems}

One of the most puzzling aspects of the $\beta$ Cep phenomenon is the well-established fact that the pulsations can decay in a time span of a few years. The most famous of these cases are Spica and 16 Lac. In the former case, the low spherical harmonic order pulsations which were observable in the radial velocities and photometry completely disappeared in a period of less than 10 years. However, high-order oscillations are still present (Smith 1985). In 16 Lac the oscillations have not completely disappeared. Both stars are close binaries, which prompted Balona (1985) to suggest that the reason for the decay in amplitude is due to a changing geometrical aspect caused by precession.

An even more tantalizing case where pulsations were seen to grow from zero amplitude in less than two years has recently been reported for the Be star $27 \mathrm{CMa}$ 
(Balona \& Rozowsky, 1991). This star is unique in two respects, being the only one in which $\beta$ Cep pulsations have suddenly appeared, and the only well-studied Be star which is also a $\beta$ Cep variable. An earlier example of a $\mathrm{Be} / \beta$ Cep star is 19 Mon (Balona \& Engelbrecht 1979, Balona et al. 1992), but the $H \alpha$ emission in this star is very weak indeed and may possibly be attributed to the pulsation. Another chapter in the intriguing $\mathrm{Be} / \beta$ Cep story was recently revealed by Mathias et al. (1991) who found that $\beta$ Cep itself had developed strong emission in $H \alpha$. Henrichs et al. (this volume) suggest that the development of emission could be related to a variable magnetic field.

These observations constitute a considerable challenge to our understanding of the driving mechanism. At this stage it is probably to early to address these issues as much work still needs to be done to make sure that the driving mechanism as we now understand it is indeed the correct one.

\section{Other B-type variables}

It has been know for some time that $\beta$ Cep stars are by no means the only intrinsically variable B-type stars. The development of high-resolution spectroscopy led to the discovery of the 53 Per stars, a group of sharp-lined stars characterized by low-order line profile variations with a period of a day or longer. Little spectroscopic work has been done on these stars, though some photometrically variable stars have been tentatively classified as members of this group or the group which has recently been termed "slowly pulsating B stars" (Waelkens, 1991).

A group which seems to be physically distinct is characterized by the prototype, the Be star $\zeta$ Oph. These are rapidly rotating stars showing high-order line profile variations of short period. These are not confined to Be stars, a counterexample being the non-Be star Spica (Smith 1985). Although the period between consecutive "moving bumps" in the line profile is measured in hours, the period in a frame of reference rotating with the star is very long and could even be infinite (Balona 1990). If this is a pulsation, it cannot be due to $p$-modes.

Finally, there is a third group which consists only of Be stars and which exhibits strictly periodic photometric variability and low-order line profile variations. These have been called $\lambda$ Eri variables, after the prototype. A statistical analysis shows that the period is not significantly different from the expected rotational period of the star (Balona 1990) and that the most likely cause of the variability is a nonuniform surface brightness distribution. Very often the light curve is of double-wave form which is phase locked from season to season and is highly suggestive of a magnetic dipole configuration (Balona et al., 1991). This type of variation is somehow connected with the Be phenomenon in a manner which is not yet understood. The discovery of $\lambda$ Eri stars in the SMC cluster NGC 330 (Balona 1992a) shows that if these stars are pulsating, the mechanism must be different from the one causing $\beta$ Cep pulsations because the metal abundance in NGC 330 is very low. 
Calculations show that $g$-modes in B stars are not excited with the current opacities, though the extreme sensitivity of the pulsation on small details of the opacity greatly diminishes the predictive power of the models. The most promising explanation for exciting $g$-modes is the mechanism originally advocated by Osaki (1974) and refined by Lee \& Saio (1986). In this mechanism overstable modes in the convective core penetrate to the envelope where they are seen as $g$-modes. The periods of these modes should be closely related to the rotation period of the core. A prediction of this theory is that the oscillations should not be produced if the convective core is too small. Further progress needs to be made in defining the predicted instability strip before the hypothesis can be put to the test.

\section{Conclusions}

The hypothesis that $\beta$ Cep pulsations are driven by the classical $\kappa$-mechanism involving the ionization zone of metals is strongly supported by the result that no such stars are found in metal-poor systems such as the two young clusters in the LMC (Balona 1992b). However, the prediction that only modes with periods close to that of the fundamental radial mode should be excited presents a problem which needs a more careful study both observationally and theoretically.

It is clear that while this mechanism may well resolve the problem of the $\beta$ Cep stars, it cannot explain the $\lambda$ Eri stars which are found in the metal-poor cluster NGC 330. At present, the models do not seem to be able to drive $g$-modes found in $53 \mathrm{Per}$ and $\zeta \mathrm{Oph}$ stars, though the latest work indicates that these may be excited by small changes in the opacities. The most pressing need at the moment are predictions of the expected limits of instability and modes of pulsation for both the $\kappa$-mechanism and by the Osaki mechanism so that these can be compared to observations.

\section{References:}

Balona, L.A., 1985. Mon. Not. R. astr. Soc., 217, 17p.

Balona, L.A., 1990. Mon. Not. R. astr. Soc., 245, 92.

Balona, L.A., 1992a. Mon. Not. R. astr. Soc., in press.

Balona, L.A., 1992b. Mon. Not. R. astr. Soc., submitted.

Balona, L.A., Cuypers, J. \& Marang, F., 1992. Astron. Astrophys. Suppl., 92, 533.

Balona, L.A. \& Engelbrecht, C.A., 1979. Mon. Not. R. astr. Soc., 189, 171.

Balona, L.A. \& Jerzykiewicz, M., 1992. SAAO Circ., submitted.

Balona, L.A. \& Feast, M.W., 1975. Mon. Not. R. astr. Soc., 172, 191.

Balona, L.A. \& Rozowsky, J., 1991. Mon. Not. R. astr. Soc., 251, 66p.

Balona, L.A. \& Shobbrook, R.R., 1984. Mon. Not. R. astr. Soc., 211, 375.

Balona, L.A., Sterken, C. \& Manfroid, J., 1991. Mon. Not. R. astr. Soc., 252, 93.

Cox, A.N. \& Morgan, S.M., 1990. Confrontation between Stellar Pulsation and Evolution, p. 293, eds Cacciari, C. \& Clementini, G., Astr. Soc. Pacif., San Francisco.

Cox, A.N., Morgan, S.M., Rogers, F.J. \& Iglesias, C.A., 1992. Astrophys. J., in press.

Engelbrecht, C.A., 1986. Mon. Not. R. astr. Soc., 223, 189. 
Grebel, E.K., Richtler, T. \& de Boer, K.S., 1992. Astr. Astrophys., 254, L5.

Iglesias, C. \& Rogers, F.J., 1991. Astrophys. J., 371, L73.

Jakate, S.M., 1980. Astr. Astrophys., 84, 374.

Jerzykiewicz, M. \& Sterken, C., 1992. In preparation.

Kiriakidis, M., El Eid, M.F. \& Glatzel, W., 1992. Mon. Not. R. astr. Soc., 255, 1 p.

Lee, U. \& Saio, H., 1986. Mon. Not. R. astr. Soc., 221, 365.

Mathias, P., Gillet, D. \& Kaper, L., 1991. in Proc. ESO Workshop Rapid variability of

OB-Stars: Nature and Diagnostic Value, p. 193 ed. D. Baade, ESO, Garching.

Millward, C.G. \& Walker, G.A.H., 1985. Astrophys. J. Suppl., 57, 63.

Moskalik, P. \& Dziembowski, W.A., 1992. Astr. Astrophys., 256, L5.

Osaki, Y., 1974. Astrophys. J., 189, 469.

Rozsnayi, B.F., 1989. Astrophys. J., 341, 414.

Shobbrook, R.R., 1979. Changing Trends in Variable Star Research, IAU Coll. 46, p.512 eds. Bateson, F.M., Smak, J. \& Urch, I.H., Univ. Waikato, Hamilton, New Zealand.

Shobbrook, R.R., 1983. Mon. Not. R. astr. Soc., 204, 47p.

Shobbrook, R.R., 1985. Mon. Not. R. astr. Soc., 214, 33.

Smith, M.A., 1985. Astrophys. J., 297, 206.

Waelkens, C., 1991. Astr. Astrophys., 246, 453.

Waelkens, C. \& Rufener, F., 1988. Astr. Astrophys., 201, L5.

Waelkens, C., Van den Abeele, K. \& Van Winckel, H., 1991. Astr. Astrophys., 251, 69.

Wolff, S.C., 1990. Astr. J., 100, 1994.

\section{Discussion}

Moskalik: I understand that W. Dziembowski, using the latest OPAL opacities which include spin-orbit interaction, now finds that the first overtone is unstable and that the minimum metal abundance for instability is somewhat lower than previously obtained.

Cox: New opacities from OPAL and OP show that doubling the $\mathrm{Z}$ is no longer necessary. $\beta$ Cep stars can now pulsate with $Z=0.03$ (using OPAL but not OP). Unfortunately the $Z=0.03 \mathrm{OP}$ opacities that are a bit smaller than OPAL do not give pulsation. Anyway, large $\mathrm{Z}$ is no longer needed, but at Los Alamos we do not get pulsation at $\mathrm{Z}=0.02$.

Walker: The Galactic $\beta$ Cep stars have spectral types B0-B3. Do the B-type stars in the three Magellanic Cloud clusters you observed have spectral types this early?

Balona: The few early-type giants for which spectral types are available do indeed fall in this range. 